TRANSACTIONS OF THE

AMERICAN MATHEMATICAL SOCIETY

Volume 275, Number 1, January 1983

\title{
THE SPECTRUM OF A RIEMANNIAN MANIFOLD WITH A UNIT KILLING VECTOR FIELD
}

BY

DAVID D. BLEECKER

\begin{abstract}
Let $(P, g)$ be a compact, connected, $C^{\infty}$ Riemannian $(n+1)$-manifold $(n \geqslant 1)$ with a unit Killing vector field with dual 1 -form $\eta$. For $t>0$, let $g_{t}=t^{-1} g$ $+\left(t^{n}-t^{-1}\right) \eta \otimes \eta$, a family of metrics of fixed volume element on $P$. Let $\lambda_{1}(t)$ be the first nonzero eigenvalue of the Laplace operator on $C^{\infty}(P)$ of the metric $g_{t}$. We prove that if $d \eta$ is nowhere zero, then $\lambda_{1}(t) \rightarrow \infty$ as $t \rightarrow \infty$. Using this construction, we find that, for every dimension greater than two, there are infinitely many topologically distinct compact manifolds for which $\lambda_{1}$ is unbounded on the space of fixed-volume metrics.
\end{abstract}

0. Introduction. Let $P$ be a compact, oriented, connected $C^{\infty}(n+1)$-manifold $(n \geqslant 1)$ with Riemannian metric $g$. We suppose $(P, g)$ has a nowhere zero Killing vector field $\xi$. By replacing $g$ by $g(\xi, \xi)^{-1} g$, we can (and do) assume $g(\xi, \xi)=1$, henceforth. Let $\eta$ be the 1 -form dual to $\xi ; \eta(\cdot) \equiv g(\xi, \cdot)$. For $t>0$, set $g_{t} \equiv t^{-1} g+$ $\left(t^{n}-t^{-1}\right) \eta \otimes \eta$, the coefficients being chosen so that the volume element is independent of $t$. When $(P, g)$ is also assumed to be homogeneous, Muto and Urakawa [6], Tanno [7] and Urikawa [8] have proved (in certain cases) that the first eigenvalue $\lambda_{1}(t)$ of the Laplace operator $\Delta_{t}$ of $g_{t}$ goes to $\infty$ as $t \rightarrow \infty$, even though $\operatorname{vol}\left(g_{t}\right)$ is constant. In this way, the question posed by Berger [1], on whether $\lambda_{1}$ is bounded for metrics of fixed volume, is answered negatively. Here we drop the assumption (among others) of homogeneity, and establish that $\lambda_{1}(t) \rightarrow \infty$ when $t \rightarrow \infty$, under the less restrictive condition that $d \eta$ is nowhere 0 . In this setting, the proof that $\lambda_{1}(t) \rightarrow \infty$ is quite different and does not require any facts about spherical representations, root systems of semisimple Lie algebras, etc.

Let $L_{\xi}: C^{\infty}(P) \rightarrow C^{\infty}(P)$ denote Lie differentiation with respect to $\xi$, and let $L_{\xi}^{2} \equiv L_{\xi} \circ L_{\xi}$. In $\S 2$, we prove that if $u \in C^{\infty}(P)$ is an eigenfunction of the Laplace operator $\Delta$ (for $g$ ), say $\Delta u=\lambda u(\lambda>0)$, and $L_{\xi}^{2} u=-k^{2} u(k \geqslant 0)$, then $\lambda-k^{2} \geqslant 0$. Equality occurs iff $d \eta=0$ and each integral submanifold of $\xi^{\perp} \equiv\{X \in T P \mid g(\xi, X)$ $=0\}$ meets each integral curve of $\xi$ in equally spaced points of spacing an integral multiple of $2 \pi k^{-1}$. In the case $d \eta$ is nowhere zero (i.e., $|d \eta|>0$ ), we prove that there is a constant $C>0$ (independent of $\lambda$ and $k$ ) such that $\lambda-k^{2} \geqslant C k$, and $\lambda-k^{2} \rightarrow \infty$ as $\lambda \rightarrow \infty$, which is used in proving $\lambda_{1}(t) \rightarrow \infty$ as $t \rightarrow \infty$.

In $\S 3$, we examine more closely the case where $\xi$ is the generator of a free $U(1)$ (circle) action. Then $\pi: P \rightarrow P / U(1) \equiv M$ is a principal $U(1)$-bundle and $g$ is a

Received by the editors February 2, 1982.

1980 Mathematics Subject Classification. Primary 53C20.

Key words and phrases. Laplacian, Riemannian manifold, Killing vector field, spectrum.

(C) 1983 American Mathematical Society 0002-9947/81/0000-0349/\$03.25 
metric invariant under the action, as in Kaluza-Klein unified field theory; see [2]. Note that $g$ determines a metric on $M$ and a connection 1 -form $i \eta$ on $P$, and vice versa. Equality occurs in $\lambda-k^{2} \geqslant 0$ iff the connection is flat and the holonomy group is cyclic of order $N$ for some $N$ dividing $k$, necessarily an integer. When $N=1,(P, g)$ is isometric to a Riemannian product of $M$ with $U(1)$. We also prove that the condition $|d \eta|>0$ is generic among $U(1)$-invariant metrics $g$ on $P$ with $g(\xi, \xi)=1$, provided $\operatorname{dim} M \geqslant 4$. Using this, we have that $\lambda_{1}$ is unbounded on the space of fixed volume metrics on any manifold of the form $M \times S^{1}$ where $M$ is any compact manifold with $\operatorname{dim} M \geqslant 4$. Even in dimensions 3 and 4 , we find infinitely many topologically distinct, compact, connected manifolds with $\lambda_{1}$ unbounded for fixed volume.

In $\S 4$, we pose some open problems. The results of $\$ 3$ have been generalized to the case of principal bundles with arbitrary compact groups. Indeed, this is the subject of the author's paper, Harmonic analysis on the space-time-charge continuum, to appear in the International Journal of Physics. In a physical context, the results of this paper suggest that in a generic universe, one cannot find charged particles of arbitrarily small mass. The author thanks Bob Reilly and Joel Weiner for helpful conversations in the development of the current paper.

1. Preliminaries. Recall that $\Delta$ is the Laplacian for functions on the $(n+1)$ manifold $P$ with metric $g$ for which $\xi$ is a unit Killing vector field with dual $\eta$. let $0=\lambda_{0}<\lambda_{1}<\lambda_{2}<\cdots$ be the distinct eigenvalues of $\Delta$ with corresponding eigenspaces $V\left(\lambda_{i}\right)$. Since $\xi$ is Killing for $g$ (and hence divergence-free), Lie differentiation $L_{\xi}$ is a skew-symmetric transformation of $C^{\infty}(P)$ with respect to the $L^{2}$ inner product. Indeed, $0=\int L_{\xi}\left(u v \mu_{g}\right)=\int\left(L_{\xi} u\right) v \mu_{g}+\int u\left(L_{\xi} v\right) \mu_{g}+\int u v L_{\xi}\left(\mu_{g}\right)$, and the last integrand vanishes. Also, since $\xi$ is Killing, it commutes with $\Delta$ and so $-L_{\xi}^{2} \mid V\left(\lambda_{i}\right)$ is a symmetric linear transformation of $V\left(\lambda_{i}\right)$ with nonnegative eigenvalues. For $k \geqslant 0$, let $C_{k} \equiv\left\{u \in C^{\infty}(P) \mid-L_{\xi}^{2} u=k^{2} u\right\}$. Then we have the orthogonal decomposition $V\left(\lambda_{i}\right)=\bigoplus_{k} C_{k} \cap V\left(\lambda_{i}\right)$, where all but finitely many summands are 0 .

Lemma 1.1. As in the introduction, let $g_{t} \equiv t^{-1} g+\left(t^{n}-t^{-1}\right) \eta \otimes \eta$ with Laplace operator $\Delta_{t}$. Then $\Delta_{t}=t \Delta+\left(t-t^{-n}\right) L_{\xi}^{2}$ and the spectrum of $\Delta_{t}$ is

$$
\left\{t \lambda_{i}-\left(t-t^{-n}\right) k^{2} \mid C_{k} \cap V\left(\lambda_{i}\right) \neq 0, k, i \geqslant 0\right\} .
$$

Proof. Recall that a Killing vector field has a projection of constant length on any geodesic. Since $\xi$ is Killing relative to $g_{t}$ (for all $t>0$ ), a geodesic of $g_{t}$ which is initially orthogonal to $\xi$ remains orthogonal. Since the distribution $\xi^{\perp} \equiv\{X \in T P \mid$ $\left.g_{t}(X, \xi)=0\right\}$ is independent of $t$ and $g_{t}=t^{-1} g$ on $\xi^{\perp}$, the curves tangent to $\xi^{\perp}$ which locally minimize arclength relative to $g$ with respect to variations in $\xi^{\perp}$, do the same relative to $g_{t}$. Thus, the geodesics of $g$ tangent to $\xi^{\perp}$ are also geodesics of $g_{t}$. Also, since $g_{t}(\xi, \xi)$ is constant, any geodesic of $g_{t}$ initially tangent to $\xi$ remains tangent (i.e., the integral curves of $\xi$ are geodesics of $g_{t}$ ). Let $p \in P$ and suppose $e_{1}, \ldots, e_{n}, e_{n+1} \equiv \xi_{p}$ is o.n. frame relative to $g$ at $p$. Then $\left(\sqrt{t} e_{1}, \ldots, \sqrt{t} e_{n}, \sqrt{t}^{-n} \xi\right)$ is 
an o.n. frame relative to $g_{t}$. For $u \in C^{\infty}(P)$, we have (where exp is the exponential map at $p$ ) at $s=0$

$$
\begin{aligned}
-\Delta_{t} u(p) & =\sum_{1}^{n} \frac{d^{2}}{d s^{2}} u\left(\exp \left(s \sqrt{t} e_{i}\right)\right)+\frac{d^{2}}{d s^{2}} u\left(\exp \left(s \sqrt{t}-n \xi_{p}\right)\right) \\
& =t \sum_{1}^{n+1} \frac{d^{2}}{d s^{2}} u\left(\exp \left(s e_{i}\right)\right)-\left(t-t^{-n}\right) \frac{d^{2}}{d s^{2}} u(\exp (s \xi)) \\
& =-t \Delta u(p)-\left(t-t^{-n}\right) L_{\xi}^{2} u(p) .
\end{aligned}
$$

For $u \in C_{k} \cap V\left(\lambda_{i}\right)$, we then have $\Delta_{t} u=\left[t \lambda_{i}-\left(t-t^{-n}\right) k^{2}\right] u$. The span of the spaces $C_{k} \cap V\left(\lambda_{i}\right)$ as $i$ and $k$ vary is dense in $C^{\infty}(P)$, since it includes each $V\left(\lambda_{i}\right)$. Hence, the various $u \in C_{k} \cap V\left(\lambda_{i}\right)$ form a complete set of eigenfunctions, and the spectrum of $\Delta_{t}$ is as stated.

REMARK. Let $k_{i}$ be the largest $k$ such that $C_{k} \cap V\left(\lambda_{i}\right) \neq 0$. For $t \geqslant 1$, we have $t-t^{-n} \geqslant 0$, and so $\lambda_{1}(t)=\min \left\{t \lambda_{i}-\left(t-t^{-n}\right) k_{i}^{2} \mid i=1,2,3, \ldots\right\}$. Since $t \lambda_{i}-$ $\left(t-t^{-n}\right) k_{i}^{2}=t\left(\lambda_{i}-k_{i}^{2}\right)+t^{-n} k_{i}^{2}$, we have $\lambda_{1}(t) \rightarrow \infty$ as $t \rightarrow \infty$, if $\lambda_{i}-k_{i}^{2} \geqslant \varepsilon>0$ for some $\varepsilon$ independent of $i \geqslant 1$. In what follows, we develop the machinery for the proof in $\S 2$ that $\lambda_{i}-k_{i}^{2} \geqslant \varepsilon>0$ (indeed, $0<\lambda_{i}-k_{i}^{2} \rightarrow \infty$ ) when $d \eta$ is nowhere 0 .

For $p \in P$, let $s \mapsto p(s)$ be the integral curve of $\xi$ through $p ; p(0)=p,-\infty<s<$ $\infty$. Let

$$
\mathbf{C}_{k} \equiv\left\{\alpha \in C^{\infty}(P, \mathbf{C}) \mid \alpha(p(s))=\exp (-i k s) \alpha(p) \text { for all } p \in P\right\} .
$$

Note that $\alpha \in \mathbf{C}_{k}$ implies $L_{\xi} \alpha=-i k \alpha$ and $-L_{\xi}^{2} \alpha=k^{2} \alpha$, whence $\operatorname{Re}(\alpha)$ and $\operatorname{Im}(\alpha) \in$ $C_{k}$. Also $L_{\xi} \alpha=-i k \alpha$ implies, for $p \in P$, that $d \alpha(p(s)) / d s=-i k \alpha(p(s))$, whence $\alpha(p(s))=\alpha(p(0)) e^{-i k s}=e^{-i k s} \alpha(p)$ (i.e., $\left.\alpha \in \mathbf{C}_{k}\right)$.

LEMMA 1.2. For $k>0$, the map Re: $\mathrm{C}_{k} \rightarrow C_{k}$ is an isomorphism with inverse $u \mapsto u+i v$ where $v \equiv k^{-1} L_{\xi} u$. Moreover, for $u+i v \in \mathbf{C}_{k}$, $v$ is the pullback of $u$ under the isometry $p \mapsto p(\pi / 2 k)$.

Proof. For $u \in C_{k}$ and $v \equiv k^{-1} L_{\xi} u$, we have $L_{\xi}(u+i v)=L_{\xi} u+i L_{\xi} v=k v-$ $i k u=-i k(u+i v)$, whence $u+i v \in \mathbf{C}_{k}$. For $u+i v \in \mathbf{C}_{k}, u(p(\pi / 2 k))=$ $\operatorname{Re}[\alpha(p(\pi / 2 k))]=\operatorname{Re}[\exp (-i \pi / 2) \alpha(p)]=\operatorname{Re}[-i \alpha(p)]=v(p)$.

Every $X \in T_{p} P$ has a unique decomposition $X=X^{H}+X^{V}$ corresponding to $T_{p} P=\mathbf{R} \xi \oplus \xi^{\perp}$. Explicitly, $X^{V}=\eta(X) \xi$ and $X^{H}=X-\eta(X) \xi$. For any $q$-form $\varphi$ on $P$, we define the $q$-form $\varphi^{H}$ by $\varphi^{H}\left(X_{1}, \ldots, X_{q}\right) \equiv \varphi\left(X_{1}^{H}, \ldots, X_{q}^{H}\right)$. Let $\langle$,$\rangle be the$ inner product induced by $g$ on the space of $\mathbf{R}$-valued forms on $T_{p} P$. Note that $\langle$, extends to $\mathrm{C}$-valued forms via $\langle\beta, \gamma\rangle \equiv\langle\operatorname{Re}(\beta), \operatorname{Re}(\gamma)\rangle+\langle\operatorname{Im}(\beta), \operatorname{Im}(\gamma)\rangle$. We write $|\beta|^{2} \equiv\langle\beta, \beta\rangle$. For a $\mathrm{C}$-valued 1-form $\varphi$, we have $\varphi=\varphi^{H}+\varphi^{V}$ where $\varphi^{V}(X)=\varphi\left(X^{V}\right)=\varphi(\eta(X) \xi)=\eta(X) \varphi(\xi)$. Moreover, $\left\langle\varphi^{H}, \varphi^{V}\right\rangle=0$, so that $|\varphi|^{2}$ $=\left|\varphi^{H}\right|^{2}+\left|\varphi^{V}\right|^{2}$. Finally, we define $\mathbf{C}_{k}\left(\lambda_{i}\right)(k>0)$ to be the space of all $\alpha=u+i v$ $\in \mathbf{C}_{k}$ such that $\Delta u=\lambda_{i} u$ (and hence $\Delta v=\lambda_{i} v$ as well, since $v$ is the pullback of $u$ by an isometry by Lemma 1.2).

Throughout the remainder of this paper, all integrals are over $P$ with respect to the volume element of $g$. 
LEMMA 1.3. For $\alpha \in \mathbf{C}_{k}\left(\lambda_{i}\right)$ with $\int|\alpha|^{2}=1, \int\left|d \alpha^{V}\right|^{2}=k^{2}$ and $\int\left|d \alpha^{H}\right|^{2}=\lambda_{i}-$ $k^{2}$.

Proof. If $\alpha=u+i v$, then $\lambda_{i}=\lambda_{i} \int|\alpha|^{2}=\lambda_{i} \int\left(u^{2}+v^{2}\right)=\int(u \Delta u+v \Delta v)=$ $\int\left(|d u|^{2}+|d v|^{2}\right)=\int|d \alpha|^{2}=\int\left|d \alpha^{H}\right|^{2}+\int\left|d \alpha^{V}\right|^{2}$. Since $d \alpha^{V}=d \alpha(\xi) \eta=\left(L_{\xi} \alpha\right) \eta$ $=-i k \alpha \eta$, we have $\int\left|d \alpha^{V}\right|^{2}=\int|i k \alpha \eta|^{2}=k^{2} \int|\alpha|^{2}=k^{2}$.

For any $q$-form $\varphi$ on $P$, we introduce the notation $D \varphi=(d \varphi)^{H}$.

LEMma 1.4. For $\alpha \in \mathbf{C}_{k}$, we have $D(D \alpha)=i k \alpha d \eta$, and $D \eta=d \eta$.

Proof. We have $L_{\xi} \eta=L_{\xi}(g(\xi, \cdot))=\left(L_{\xi} g\right)(\xi, \cdot)+g\left(L_{\xi} \xi, \cdot\right)=0$, and so $0=$ $\left.\left.L_{\xi} \eta=d(\eta\lrcorner \xi\right)+(d \eta)\right\lrcorner \xi=d \eta(\xi, \cdot)$, whence $d \eta=D \eta$. Now $D \alpha=(d \alpha)^{H}=d \alpha-$ $d \alpha(\xi) \eta=d \alpha-\left(L_{\xi} \alpha\right) \eta=d \alpha+i k \alpha \eta$. Thus, $d(D \alpha)=i k d \alpha \wedge \eta+i k \alpha d \eta$. Since $\eta^{H}$ $=0$ and $(d \eta)^{H}=d \eta, D(D \alpha)=i k \alpha d \eta$.

2. Main results. If $d \eta=0$, then the distribution $\xi^{\perp}$ is integrable and any maximal connected integral submanifold $Q$ is a complete, totally geodesic, immersed submanifold of $P$; the proof of Lemma 1.1 shows $Q$ to be totally geodesic. The map $f$ : $Q \times \mathbf{R} \rightarrow P$ given by $f(q, s)=q(s)$ is a local isometry, where $Q \times \mathbf{R}$ is given the product metric $(g \mid Q) \times d s^{2}$. Since $Q \times \mathbf{R}$ is complete with this metric, $f$ is then a Riemannian covering; see [5]. In particular, $f$ is onto. If $\varphi(s): P \rightarrow P$ is the map $p \mapsto p(s)$, then $\varphi(s)(Q)$ is an integral submanifold of $\xi^{\perp}$, and since $f$ is onto, all integral submanifolds of $\xi^{\perp}$ are of the form $\varphi(s)(Q), s \in \mathbf{R}$. The "holonomy group" of $\eta$ is defined as $H(\eta) \equiv\{s \in \mathbf{R} \mid \varphi(s)(Q)=Q\}$, which is independent of the choice of $Q$. Note that $H(\eta) \neq 0$, for otherwise $f$ would be 1-1 and hence an isometry, but $Q \times \mathbf{R}$ is noncompact unlike $P$. There are examples where $H(\eta)$ is not discrete; consider flat tori.

Recall that $k_{i} \equiv \max \left\{k \mid C_{k} \cap V\left(\lambda_{i}\right) \neq 0\right\}=\max \left\{k \mid \mathbf{C}_{k}\left(\lambda_{i}\right) \neq 0\right\}$.

THEOREM 2.1. For each $i \geqslant 1$, we have $k_{i}^{2} \leqslant \lambda_{i}$. If $k_{i}^{2}=\lambda_{i}$, then $d \eta=0$ and $H(\eta)$ is discrete with generator being an integral multiple of $2 \pi k_{i}^{-1}$. Conversely, if $d \eta=0$ and $H(\eta)$ has generator $2 \pi k^{-1}$, then $N^{2} k^{2}$ is an eigenvalue of $\Delta$ for any integer $N \geqslant 0$, and $\mathbf{C}_{N k}\left(N^{2} k^{2}\right) \neq 0$.

Proof. Let $\alpha \in \mathbf{C}_{k_{i}}\left(\lambda_{i}\right)$ with $\alpha \neq 0$. Lemma 1.3 implies $k_{i}^{2} \leqslant \lambda_{i}$. Assume $k_{i}^{2}=\lambda_{i}$. Then Lemma 1.3 implies $D \alpha=0$, and Lemma 1.4 gives $0=D(D \alpha)=i k_{i} \alpha d \eta$. Since $\alpha$ is a (complex) eigenfunction of $\Delta$ and $\alpha \neq 0$, the support of $\alpha$ is all of $P$; by unique continuation $\alpha$ does not vanish on an open set; see [3]. Hence, $\alpha d \eta=0$ implies $d \eta=0$. Since $d \alpha \mid \xi^{\perp}=0, \alpha$ is constant on any maximal connected integral submanifold $Q$ of $\xi^{\perp}$. For any $s \in \mathbf{R}$, note that $\alpha(\varphi(s)(Q))=\exp \left(-i k_{i} s\right) \alpha(Q)$. This not only proves that $|\alpha| \equiv$ const, but also that $\varphi(s)(Q)=Q$ (i.e., $s \in H(\eta)$ ) implies $k_{i} s=2 \pi N$ for some integer $N$. Thus, $H(\eta)$ is discrete with generator being an integer multiple of $2 \pi k_{i}^{-1}$.

Conversely, if $d \eta=0$ with $H(\eta)$ discrete with generator $2 \pi k^{-1}$, let $Q$ be a maximal connected integral submanifold of $\xi^{\perp}$ and define $\alpha: P \rightarrow \mathbf{C}$ by $\alpha(q(s))=$ $\exp (-i k s)$ for $q \in Q$. Note that $\alpha$ is well defined, since $q(s)=q^{\prime}\left(s^{\prime}\right)$ implies $q^{\prime}=q\left(s-s^{\prime}\right)$; and so $s-s^{\prime} \in H(\eta), s-s^{\prime} \in 2 \pi k^{-1} \mathbf{Z}$, and $\exp (i k s)=\exp \left(i k s^{\prime}\right)$. 
Note that $\alpha$ is constant on each of the totally geodesic submanifolds $\varphi(s)(Q)$, and the integral curves of $\xi$ are geodesics orthogonal to these. Thus, for any integer $N \geqslant 0, \Delta\left(\alpha^{N}\right)=-L_{\xi}^{2}\left(\alpha^{N}\right)$. Since $L_{\xi} \alpha^{N}=-i N k \alpha^{N}$, we have $\Delta\left(\alpha^{N}\right)=N^{2} k^{2} \alpha^{N}$ and so $0 \neq \alpha^{N} \in \mathbf{C}_{N k}\left(N^{2} k^{2}\right)$.

THEOREM 2.2. If $d \eta$ is nowhere zero, then there is a constant $C>0$ such that for $i \geqslant 0, \lambda_{i}-k_{i}^{2} \geqslant C k_{i}$ and $\lambda_{i}-k_{i}^{2} \rightarrow \infty$ as $i \rightarrow \infty$.

Proof. Since $P$ is compact, $\min |d \eta|^{2}>0$. Let $\alpha \in \mathbf{C}_{k}\left(\lambda_{i}\right)$ with $\int|\alpha|^{2}=1$. Then $k^{2} \min |d \eta|^{2} \leqslant \int k^{2}|d \eta|^{2}|\alpha|^{2}=\int|i k \alpha d \eta|^{2}=\int\langle D(D \alpha), i k \alpha d \eta\rangle$ by Lemma 1.4. Let $\delta: \Lambda^{q}(P, \mathbf{C}) \rightarrow \Lambda^{q-1}(P, \mathbf{C})$ be the codifferential operator, adjoint to $d$, on $q$-forms; recall $\delta=(-1)^{n q+n+q} * d *$, where $*$ is the Hodge star operator relative to $g$ and $\mu_{g}$, and $\operatorname{dim} P=n+1$. Dividing by $k$, we then obtain (since $d \eta=$ $\left.(d \eta)^{H}\right) \quad k \min |d \eta|^{2} \leqslant \int\langle d(D \alpha), i \alpha d \eta\rangle=\int\langle D \alpha, i \delta(\alpha d \eta)\rangle$. Now $\delta(\alpha d \eta)=$ $(-1)^{n} * d *(\alpha d \eta)=(-1)^{n} *(d \alpha \wedge * d \eta)+\alpha \delta d \eta$. Since $d \eta^{H}=d \eta$, we have $* d \eta=$ $\beta \wedge \eta$ for some form $\beta$, whence $d \alpha \wedge * d \eta=(D \alpha-i k \alpha \eta) \wedge \beta \wedge \eta=D \alpha \wedge \beta \wedge \eta$ $=D \alpha \wedge * d \eta$. Since $* d \eta$ is real,

$$
\begin{aligned}
|D \alpha \wedge * d \eta|^{2} & =|\operatorname{Re}(D \alpha) \wedge * d \eta|^{2}+|\operatorname{Im}(D \alpha) \wedge * d \eta|^{2} \\
& \leqslant\left[|\operatorname{Re}(D \alpha)|^{2}+|\operatorname{Im}(D \alpha)|^{2}\right]|* d \eta|^{2}=|D \alpha|^{2}|d \eta|^{2}
\end{aligned}
$$

Thus

$$
\begin{aligned}
& |\langle D \alpha, i \delta(\alpha d \eta)\rangle| \leqslant \mid\langle D \alpha, i *(D \alpha \wedge * d \eta\rangle|+|\langle D \alpha, \alpha \delta d \eta\rangle| \\
& \quad \leqslant|D \alpha||D \alpha \wedge * d \eta|+\left|\left\langle D \alpha, \alpha(\delta d \eta)^{H}\right\rangle\right| \leqslant|D \alpha|^{2}|d \eta|+|D \alpha||\alpha|\left|\Delta^{H} \eta\right|
\end{aligned}
$$

where $\Delta^{H} \eta \equiv[(\delta d+d \delta)(\eta)]^{H}=(\delta d \eta)^{H}$, since $\delta \eta=-\operatorname{div} \eta=0$. Hence, we obtain

$$
\begin{aligned}
k \min |d \eta|^{2} & \leqslant \int|D \alpha|^{2}|d \eta|+\int|D \alpha||\alpha| \Delta^{H} \eta \mid \\
& \leqslant \max |d \eta| \int|D \alpha|^{2}+\max \left|\Delta^{H} \eta\right|\left[\int\left|\alpha^{2}\right| \int|D \alpha|^{2}\right]^{1 / 2} .
\end{aligned}
$$

Thus, setting $k=k_{i}$ and using Lemma 1.3 , we obtain

$$
k_{i} \min |d \eta|^{2} \leqslant \max |d \eta|\left(\lambda_{i}-k_{i}^{2}\right)+\max \left|\Delta^{H} \eta\right|\left(\lambda_{i}-k_{i}^{2}\right)^{1 / 2} .
$$

Now $\lambda_{i}-k_{i}^{2} \rightarrow \infty$ as $i \rightarrow \infty$, for otherwise a subsequence $\lambda_{i_{m}}-k_{i_{m}}^{2}$ would be bounded and (*) would imply that $k_{i_{m}}$ is bounded, but $\lambda_{i_{m}} \rightarrow \infty$ and so $\lambda_{i_{m}}-k_{i_{m}} \rightarrow$ $\infty$, a contradiction. For any $\varepsilon>0$, we can find $N$ such that for $i>N,\left(\lambda_{i}-k_{i}^{2}\right)>\varepsilon^{-2}$, and so $\left(\lambda_{i}-k_{i}^{2}\right)^{1 / 2}<\varepsilon\left(\lambda_{i}-k_{i}^{2}\right)$. Then (*) yields

$$
k_{i} C_{\varepsilon} \equiv k_{i} \min |d \eta|^{2}\left[\max |d \eta|+\varepsilon \max \left|\Delta^{H} \eta\right|\right]^{-1} \leqslant \lambda_{i}-k_{i}^{2}
$$

for $i>N$. Since $d \eta \neq 0$, Theorem 2.1 implies $\lambda_{1}-k_{1}^{2}>0, \ldots, \lambda_{N}-k_{N}^{2}>0$, whence $0<C \equiv \min \left\{C_{\varepsilon}, k_{1}^{-1}\left(\lambda_{1}-k_{1}^{2}\right), \ldots, k_{N}^{-1}\left(\lambda_{N}-k_{N}^{2}\right)\right\}$ and $\lambda_{i}-k_{i}^{2} \geqslant C k_{i}$ for $i \geqslant 0$; note $\lambda_{0}=k_{0}=0$.

THEOREM 2.3. If $\xi$ is a unit Killing vector field on $(P, g)$ with dual 1-form $\eta$, and $\lambda_{1}(t)$ is the first nonzero eigenvalue of the Laplacian for $t^{-1} g+\left(t^{n}-t^{-1}\right) \eta \otimes \eta$, then $\lambda_{1}(t) \geqslant \varepsilon t$ for some $\varepsilon>0$ and $t \geqslant 1$ (e.g., $\lambda_{1}(t) \rightarrow \infty$ as $t \rightarrow \infty$ ) provided $d \eta$ is nowhere zero. 
Proof. Since $d \eta \neq 0$, we know $\lambda_{i}-k_{i}^{2}>0$ for $i \geqslant 1$, by Theorem 2.1. Since $|d \eta|>0$, we have $\lambda_{i}-k_{i}^{2} \rightarrow \infty$ as $i \rightarrow \infty$. Thus, $\varepsilon \equiv \min \left\{\lambda_{i}-k_{i}^{2} \mid i \geqslant 1\right\}$ exists and $\varepsilon>0$. By the Remark following Lemma 1.1, for $t \geqslant 1$,

$$
\lambda_{1}(t)=\min \left\{t\left(\lambda_{i}-k_{i}^{2}\right)+t^{-n} k_{i}^{2} \mid i \geqslant 1\right\} \geqslant \min \left\{t\left(\lambda_{i}-k_{i}^{2}\right) \mid i \geqslant 1\right\}=t \varepsilon .
$$

3. Principal $U(1)$-bundles. In this section, we assume that $\xi$ is the infinitesimal generator of a free $U(1)$ action (by isometries) of $(P, g)$. Thus, $\pi: P \rightarrow P / U(1) \equiv M$ is a principal $U(1)$-bundle with $i \eta$ being a $\mathscr{Q}(1)$-valued connection 1 -form on $P$; $\mathcal{U}(1) \equiv\{$ is $\mid s \in \mathbf{R}\}$. Note that $g$ induces a Riemannian metric $g_{M}$ on $M$. Recall that $\alpha \in \mathbf{C}_{k}$ when $\alpha(p(s))=\exp (-i k s) \alpha(p)$, but $p(2 \pi)=p$, whence $\mathbf{C}_{k}=0$ unless $k$ is an integer. In this setting, the holonomy group of $i \eta$, in the case $i \eta$ is flat (i.e., $d \eta=0)$, is taken to be $\exp (i H(\eta)) \subset U(1)$, where $H(\eta)$ was defined in $\S 2$. The analogue of Theorem 2.1 is clearly

THEOREM 3.1. For each $i \leqslant 1$, we have $k_{i}^{2} \leqslant \lambda_{i}$. If $k_{i}^{2}=\lambda_{i}$, then in is flat with holonomy group $\mathbf{Z}_{k} \equiv\{\exp (m 2 \pi i / k) \mid m=0,1,3, \ldots, k-1\}$ for some integer $k$ dividing $k_{i}$. Conversely, if i $\eta$ is flat with holonomy group $\mathbf{Z}_{k}$, then $N^{2} k^{2}$ is an eigenvalue of $\Delta$ for any integer $N \geqslant 0$, and $\mathbf{C}_{N k}\left(N^{2} k^{2}\right) \neq 0$.

Corollary 3.2. Assume $k_{i}^{2}=\lambda_{i}$ for some $i \geqslant 1$. Then $\pi: P \rightarrow M$ is a trivial bundle. If the holonomy group of $i \eta$ is trivial, then $(P, g)$ is isometric to $M \times U(1)$ with the product metric $g_{M} \times d s^{2}$. The holonomy group is trivial if $M$ is simply-connected, or if $k_{j}^{2}=\lambda_{j}$ for some $j \geqslant 1$ with $k_{i}$ and $k_{j}$ relatively prime.

Proof. If $k_{i}^{2}=\lambda_{i}$, then $d \eta=0$, so that the Chern class of $\pi: P \rightarrow M$ is trivial. For $U$ (1)-bundles, this implies $\pi: P \rightarrow M$ is trivial. If $Q$ is a maximal connected integral submanifold of $\xi^{\perp}$, then $\pi \mid Q:(Q, g \mid Q) \rightarrow\left(M, g_{M}\right)$ is a Riemannian $N$-fold covering where $N$ is the order of the holonomy group. When $N=1$, the map $q(s) \mapsto\left(\pi(q), e^{i s}\right)$ for $q \in Q$ defines an isometry from $P$ to $M \times U(1)$. By Theorem $3.1, N$ divides $k_{i}$ and $k_{j}$, etc.

THEOREM 3.3. If $\lambda_{1}(t)$ is the first nonzero eigenvalue of the Laplacian for $t^{-1} g+$ $\left(t^{n}-t^{-1}\right) \eta \otimes \eta$, then $\lambda_{1}(t) \rightarrow 0$ as $t \rightarrow 0$. If $|d \eta|>0$, then $\lambda_{1}(t) \rightarrow \infty$ as $t \rightarrow \infty$.

Proof. If $\Delta_{M}$ is the Laplacian for $g_{M}$, then for $f \in C^{\infty}(M)$, we have $\Delta(f \circ \pi)=$ $\left(\Delta_{M} f\right) \circ \pi$, since horizontal geodesics on $P$ project to geodesics on $M$. Let $\mu$ be the first nonzero eigenvalue of $\Delta_{M}$ and $\Delta_{M} f=\mu f$ where $f \neq 0$. Then $f \circ \pi \in \mathbf{C}_{0}(\mu)$ and Lemma 1.1 implies $\lambda_{1}(t) \leqslant t \mu$. Thus, $\lambda_{1}(t) \rightarrow 0$ as $t \rightarrow 0$. The last statement follows from Theorem 2.3.

Let $G(P)$ be the space of Riemannian metrics on $P$ for which $\xi$ is a unit Killing vector field, and let $G_{0}(P)=\{g \in G(P)|| d \eta \mid>0, \eta(\cdot) \equiv g(\xi, \cdot)\}$. We endow $G(P)$ with the topology of uniform convergence of each $k$-jet, $k=0,1,2, \ldots$

Theorem 3.4. If $\operatorname{dim} M \geqslant 4$, then $G_{0}(P)$ is open and dense in $G(P) \neq \varnothing$.

Proof. By averaging any metric on $P$ with respect to the action of the compact group $U(1)$ and dividing by the length-squared of $\xi$, we can produce a member of $G(P)$. It is clear that $G_{0}(P)$ is open in $G(P)$, and we need only establish density. 
Let $J_{q}^{r}(M) \rightarrow M$ be the bundle of $q$-jets of $r$-forms. For an $r$-form $\alpha$, we let $j_{q}(\alpha)$ : $M \rightarrow J_{q}^{r}(M)$ be the $q$-jet extension of $\alpha$. Exterior differentiation induces a bundle map $d(r, q): J_{q}^{r}(M) \rightarrow J_{q-1}^{r+1}(M)$. Select any $g \in G(P)$ and let $\eta(\cdot)=g(\xi, \cdot)$. There is a unique closed 2-form $F$ on $M$ such that $\pi^{*} F=d \eta$, since $(d \eta)^{H}=d \eta$ and $d \eta$ is invariant under the $U(1)$ action. For any $x \in M$, let $W_{x}=\left\{\sigma \in J_{1}^{1}(M)_{x} \mid d(1,1)(\sigma)\right.$ $\left.=F_{x}\right\}$ or equivalently, $W_{x}=j_{1}\left(\eta^{\prime}\right)(x)+\operatorname{ker} d(1,1)_{x}$ where $\eta^{\prime}=\tau^{*} \eta$ for some local section $\tau: U \rightarrow P$ with $x \in U$. Note that $\cup\left\{W_{x} \mid x \in M\right\} \equiv W \rightarrow M$ is a smooth subbundle of $J_{1}^{1}(M)$ of codimension $n(n-1) / 2(n=\operatorname{dim}(M))$ which is greater than $n$ for $n \geqslant 4$. By standard transversality theory, for $n \geqslant 4$, the space $S$ of 1-forms $\varphi$ on $M$ such that $j_{1}(\varphi)$ is transverse to $W$ (i.e., $d \varphi_{x} \neq F_{x}, \forall x \in M$ ) is open and dense. For any 1 -form $\varphi$ on $M$, let $g(\varphi) \in G(P)$ be defined by $g(\varphi)=\pi^{*} g_{M}+$ $\left(\eta-\pi^{*} \varphi\right) \otimes\left(\eta-\pi^{*} \varphi\right)$. Since $g(\varphi)(\xi, \cdot)=\eta(\xi)\left(\eta-\pi^{*} \varphi\right)(\cdot)$, the dual of $\xi$ relative to $g(\varphi)$ is $\eta-\pi^{*} \varphi$. If $\varphi \in S$, then $d\left(\eta-\pi^{*} \varphi\right)=\pi^{*}(F-d \varphi) \neq 0$, whence $g(\varphi) \in$ $G_{0}(P)$. Since the map $\varphi \mapsto g(\varphi)$ is continuous, the image of the dense set $S$ must meet every neighborhood of $g=g(0)$. Since $g \in G(P)$ is arbitrary, we have $G_{0}(P)$ is dense.

Corollary 3.5. Any compact $C^{\infty}$ manifold $P$ with $\operatorname{dim} P \geqslant 5$ which admits a free $U(1)$ action has a family of metrics of fixed volume for which $\lambda_{1}$ is unbounded. In particular, $P=M \times S^{1}$ for any compact $M$ with $\operatorname{dim} M \geqslant 4$ will do.

Proof. By Theorem 3.4, we have some $g \in G_{0}(P)$ (i.e., $|d \eta|>0$ ), and Theorem 3.3 applies.

An infinite collection of topologically distinct examples (where Theorem 3.3 can be applied) of dimension 3 are obtained by taking $P$ to be the o.n. frame bundle of an oriented compact Riemannian 2-manifold $M$ with curvature nowhere zero. In this case, the metric $g$ on $P$ is that one determined by the metric on $M$ and the Levi-Civita connection on $P$; $d \eta$ is then the pullback of the curvature form of $M$ (e.g., $|d \eta|>0$ ). As long as $\chi(M) \neq 0$, a metric on $M$ can be chosen so that the curvature is nowhere zero, whence $P$ admits a family of metrics of fixed volume on which $\lambda_{1}$ is unbounded. If we give $P \times S^{1}$ the product metric and extend the $U(1)$ action to $P \times S^{1}$ by letting $U(1)$ act trivially on $S^{1}$, then $d \eta$ is still nowhere zero, and Theorem 3.3 applies to the various $P \times S^{1}$ of dimension 4 .

4. Open problems. A. In the event that $\xi$ generates a free $U(1)$ action, (by Theorem 3.1) the Chern class $c(P)$ of the $U(1)$-bundle $\pi: P \rightarrow P / U(1)$ vanishes when $k_{i}^{2}=\lambda_{i}$ for some $i \geqslant 1$. Does $c(P) \neq 0$ imply $\lambda_{i}-k_{i}^{2} \geqslant \varepsilon>0$ for some $\varepsilon>0$ independent of $i$; or do we at least have $\lambda_{1}(t) \rightarrow \infty$ as $t \rightarrow \infty$ ? Note that $k_{i}^{2}<\lambda_{i}$ is not enough to obtain $\lambda_{1}(t) \rightarrow \infty$. In fact, one can construct $\xi$ on some flat torus of dimension $\geqslant 2$ such that $d \eta=0$ and $H(\eta)$ is not discrete, whence $k_{i}^{2}<\lambda_{i}$ for all $i \geqslant 1$, by Theorem 3.1. However, Berger [1] has proved that $\lambda_{1}$ is bounded on the space of flat metrics of fixed volume on a torus, whence $\lambda_{1}(t)$ is bounded.

B. The constant $C$ in the proof of Theorem 2.2 is probably not the best. Joel Weiner has shown in the case where $\xi$ generates a free $U(1)$ action and $\operatorname{dim}[P / U(1)]$ $=2$, we can take $C$ to be $\min |d \eta|$. What is the best possible $C$ in terms of $d \eta$, $c(P)$, etc? 
C. We have seen that in every dimension greater than 2 , there are infinitely many compact manifolds for which $\lambda_{1}$ is unbounded on the space of metrics of fixed volume. All our examples admit metrics with nonzero Killing fields, but we suspect that this is irrelevant. That is, we conjecture that $\lambda_{1}$ is unbounded for fixed-volume metrics on any compact $n$-manifold, $n \geqslant 3$. For a compact surface of genus $G$, there is the result $\lambda_{1} \leqslant 8 \pi(G+1)$ (for metrics of volume 1) due to Yang and Yau [9] when $G>0$; the case $G=0$ is the Hersch result [4].

\section{BIBLIOGRAPHY}

1. M. Berger, Sur les premières valeurs propres des variétés Riemanniennes, Compositio Math. 26 (1973), 129-149.

2. D. Bleecker, Gauge theory and variational principles, Global Analysis, vol. I, Addison-Wesley, Reading, Mass., 1981.

3. A. Calderón, Uniqueness in the Cauchy problem for partial differential equations, Amer. J. Math. 80 (1958), 16-36.

4. J. Hersch, Quatre propriétés isopérimétriques de membranes sphériques homogènes, C. R. Acad. Sci. Paris Sér. A-B 270 (1970), 1645-1648.

5. S. Kobayashi and K. Nomizu, Foundations of differential geometry, vol. I, Interscience, New York, 1963.

6. H. Muto and H. Urakawa, On the least positive eigenvalue of the Laplacian for compact homogeneous spaces, Osaka J. Math. 17 (1980), 471-484.

7. S. Tanno, The first eigenvalue of the Laplacian on spheres, Tôhoku Math. J. 31 (1979), 179-185.

8. H. Urakawa, On the least positive eigenvalue of the Laplacian for compact group manifolds, J. Math. Soc. Japan 31 (1979), 209-226.

9. P. Yang and S. T. Yau, Eigenvalues of the Laplacian of compact Riemann surfaces and minimal submanifolds, Ann. Scuola Norm. Sup. Pisa 7 (1980), 55-63.

Deparment of Mathematics, University of Hawail, Honolulu, HawaII 96822 